\title{
REVIEW
}

\section{Selenium and the prostate}

\author{
Wilfred N. Arnold \\ Department of Biochemistry and Molecular Biology, University of Kansas Medical Center, Kansas City, Kansas, \\ USA
}

Received $12^{\text {th }}$ February 2005.

Revised $18^{\text {th }}$ April 2005.

Published online $10^{\text {th }}$ May 2005.

\begin{abstract}
Summary
Evidence accrued over the last decade has supported an association between low serum selenium (Se) and increased incidence of prostate cancer in older men. Accordingly, questions and inquiry arose as to whether a dietary supplementation might afford protection, or delay disease progression. Indeed, a very large national study, Selenium and Vitamin E Cancer Prevention Trial (SELECT), to explore the potential benefits of a daily supplement of 200 microgram Se, or 400 I.U. of vitamin E, or both, is underway: the National Cancer Institute anticipates a total enrolment of 32,400 men and durations of 7 to 12 years for individual participants. It is remarkable that at the time of the trial's activation in 2001 nothing was known about the concentration of Se in the target organ or whether any Se in a dietary supplement found its way to the prostate gland. Later, our laboratory published the first benchmark values, which indicated that the prostate is reasonably well endowed with $\mathrm{Se}$ and that the concentration in one subject, who had undergone self-medication at 200 microgram Se per day, was about twice the average for the others. This review explores the ramifications of these and subsequent data for SELECT and other trials, together with observations on dose and general mechanisms of Se interference with the development of prostate cancer.
\end{abstract}

Key words: selenium - prostate - cancer - supplementation trials

\section{INTRODUCTION}

Selenium (Se) is an essential animal micronutrient, although the realization of its biological importance

Wilfred N. Arnold, University of Kansas Medical Center, Kansas City, Kansas 66160-7421, USA

昌warnold @kumc.edu has a relatively short history compared with other minerals. It was not until 1957 that a role for Se was found in the protection of rats and mice from necrotic liver degeneration (Schwarz and Folz 1957) and of chickens from an exudative diathesis (Schwarz et al. 1957, Patterson et al. 1957). Cultured mammalian cells were later shown to require $\mathrm{Se}$ for growth (Guilbert and Iscoe 1976, McKeehan et al. 1976). Specific enzymes such as glutathione peroxidase (Rotruck et al. 1973, Epp et al. 1983), deiodinase 
(Arthur et al. 1991) and thioredoxin reductase (Hill et al. 1997) are now known to contain essential selenocysteine residues, which are biosynthesized from dietary Se. (Stadtman 1987, 1992)

Human disorders due to Se-deficiency include Keshan disease (a cardiomyopathy) and Kashin-Beck disease (an osteoarthropathy) - both can be successfully treated with Se supplementation of the diet (Ge and Yang 1993). Hypothyroid cretinism (Vanderpas et al. 1990) requires both Se and iodine intervention (Moreno-Reyes et al. 1998). (These endemic diseases have been restricted to regions of Asia and Africa.) Links between low Se intake and ischemic heart disease (Salonen and Huttunen 1986), and with vulnerability of hepatocytes to alcohol (Aaseth et al. 1986), have been suggested. The investigations by Beck et al. $(1994,1995)$ and Beck (1997) into a human enterovirus, carried in a mouse model with a Se compromise, are the first documented reports of a specific nutritional deficiency invoking changes in a viral genome and resulting in an acquired virulence by the pathogen.

\section{Selenium intake}

Variation in the Se concentration of soils was formerly the largest influence on its status in humans because $\mathrm{Se}$ is efficiently transferred up the soil plant - animal - human food chain. This circumstance enabled epidemiological surveys which explored associations among local Se levels and health issues, including incidences of various cancers (Shamberger and Frost 1968, Clark et al. 1991). The observed differences among regions of the U.S. that were significant fifty years ago are now largely eliminated by the interstate transportation of foodstuffs. The recommended daily intake (Levander 1987, Sunde 1997) of selenium for humans is currently 60 microgram. Typical one-a-day formulations of vitamins and minerals in the U.S.A. contain a modest 20 microgram Se per tablet, but over-the-counter selenium supplements of 200 microgram Se per tablet are also readily available.

In the late 1970's and 1980's the intake of Finns was estimated at only 20-30 microgram Se per day and Se-fortification of Finnish fertilizers was started in 1984. Five years later the adult average intake stabilized at 125 microgram Se per day and by 1993 it was successfully adjusted to 80 microgram Se per day (Hartman et al. 2002). It may be too early to tell whether the general health of that population has been modified. We also note that in the recent past, patients in Europe and the U.S. on either parenteral or restricted, gastrointestinal nutrition were vulnerable to Se-deficiency (Levander 1987), but appropriate amounts of selenium are now routinely added to special diets, as exemplified by a set of neonatal cases (Lombeck et al. 1975).

\section{Toxicity}

The mixed regard for selenium in mammalian nutrition suffers from the unusual circumstance that its potential for toxicity (Levander 1972), which dates from the 1930's, preceded proof of its essentiality and indications of protective effects against particular diseases. Among experimental animals the single, oral $\mathrm{LD}_{50}$ values for sodium selenite vary between 2,300 and 13,000 microgram Se per kilogram body weight (Levander 1987). For humans, the "no observable adverse effect level" is given as 800 microgram Se per day, and the "lowest observed adverse effect level" is said to be 1,260 microgram Se per day (Yang and Zhou 1994).

In 1984, a 57-year-old New York woman fell victim to an error in pharmaceutical formulation (Jensen et al. 1984). The selenium supplement she used was labeled 150 microgram $\mathrm{Se}$, but was eventually found to contain 31,000 microgram Se per tablet (mostly as sodium selenite). She took a daily tablet for 11 weeks before the product was recalled by the distributor for "superpotency." Marked hair loss from her scalp was noted after 11 days from the start of self-medication and progressed over the next 50 days to almost total alopecia. At 25 days she noticed a white streak on the fingernail of a little finger, then experienced tenderness and swelling of the fingertip, followed by a purulent discharge from the fingernail bed and the eventual loss of the entire nail. All fingernails were eventually affected. She also suffered episodes of nausea and vomiting, bad breath, and fatigue - these symptoms were consistent with Se toxicity (Yang et al 1983). At the time she stopped the supplement, her serum contained 528 microgram Se per L; about 4 times normal for the U.S. population. (Much of the megadose of Se was supposedly excreted, but was not documented.) An editorial note (Jensen et al 1984) suggested that things may have been worse had the subject not been taking each day $2 \mathrm{~g}$ of vitamin $\mathrm{C}, 1 \mathrm{~g}$ of which she ingested simultaneously with the $\mathrm{Se}$ supplement. Vitamin C reduces selenite to elemental Se, which is poorly absorbed (Medinsky et al. 1981).

\section{Blood levels}

Traditionally, Se status has been assessed by measuring circulating Se levels. In general, serum or plasma concentrations are more sensitive to the subject's immediate selenium intake and status than are whole blood determinations, but both reach plateau values at supranutritional concentrations of Se exposure (Raich et al. 2001). There are data bases which show interesting differences among countries 
(Schaller and Schiele 1989). Sheehan and Halls (1999) found the following levels in the U.K.: for 01.4 years $=45-50$ microgram Se per L plasma; $1.5-$ 4.0 y. $=45-90 ; 4.1-16$ y. $=55-115$; adult $=70-130$. Pohl and Schneider (1991) measured Se in the plasma of 126 German males and found a mean of $78 \pm 27$ microgram per $\mathrm{L}$, and no relationship with age, between 15 and 65, was discerned.

A majority of surveys have indicated a statistically significant association between low serum or plasma Se concentration and cancer, even though the magnitudes of the reported differences are less than over-whelming. For example, Willett et al.(1983) collected samples in 1973 and then compared values for subjects who developed a cancer during the next five years [129 \pm 2 microgram Se per $\mathrm{L}$ serum; $\mathrm{n}=111]$ with controls (matched for age, race, sex, and smoking history) who remained cancer-free [136 $\pm 2 ; n=210]$. The risk of cancer for subjects in the lowest quintile of serum selenium was said to be twice that of subjects in the highest. The association with low serum selenium level was strongest for gastrointestinal and prostatic cancers. A retrospective analysis (Health Professionals FollowUp Study) of long term selenium status (measured by toenail analyses) found that men in the highest quintile for selenium concentration in 1987 had about half the risk of developing advanced prostate cancer in 1989-1994 compared with men in the lowest quintile (Yoshizawa et al. 1998). Later, a similar conclusion was based on plasma Se levels and incidence of prostate cancer (Brooks et al. 2001).

\section{Prostate cancer}

For U.S. males the probability of developing prostate cancer is presently less than $0.01 \%$ for those at age $0-40$; about $2 \%$ for $40-60 ; 14 \%$ for $60-80$; and $17 \%$ for over 80 [adapted from National Cancer Institute's SEER, March 2002]. Prostate cancer is the most common cancer among Veteran Service Men in the U.S.A. (Kim et al 1999). No age related decrease in serum or plasma $\mathrm{Se}$ for healthy men has been demonstrated, but the epidemiologic examples have encouraged a working hypothesis that supplemental Se might reduce the risk of prostate cancer in older men. Se concentration in the blood plasma (at least for sub-saturation levels) can be increased from about 70 to 170 microgram per L in 11 weeks by a daily dietary supplement of 200 microgram Se in the form of enriched yeast (Levander et al. 1983). It followed that controlled trials involving a deliberate boost in Se intake might be instructive. Based on toxicity considerations, a therapeutic intervention window of approximately 60-1,000 microgram total Se intake per day has been implied by most commentators.

\section{SELENIUM SUPPLEMENTATION TRIALS}

Men assigned to receive 200 microgram selenium daily for 4.5 years had one third the relative risk for prostate cancer compared with those assigned the placebo in a randomized, double-blind test (Nutritional Prevention of Cancer Study Group), which is commonly referenced as the Clark trial (Clark et al. 1996, 1998). It involved 980 men and 332 women, half of whom received a daily tablet containing 200 microgram Se (as enriched yeast) to test whether selenium supplementation would reduce the incidences of squamous or basal cell carcinomas of the skin. The primary hypothesis was not supported by the forthcoming data but the blinded phase of the trial was stopped early because of apparent reductions in cancer mortality and incidences (especially due to lung, colorectal, and prostate cancers) within the group receiving the Se supplement. Indications from this study, together with the retrospective findings of Yoshizawa et al. (1998) were apparently most influential in promoting a much larger Selenium and Vitamin E Cancer Prevention Trial (SELECT), which retained the Clark trial intervention level for Se at 200 microgram per day.

\section{SELECT}

The rationale and design for SELECT were presented by Klein et al. (2000), "recent analysis of secondary endpoints of several large-scale randomized prospective clinical trials for other malignancies has suggested that selenium or vitamin E may result in a decreased incidence and mortality from prostate cancer. In vitro and preclinical studies of these antioxidants support this hypothesis." Elsewhere (Hoque et al. 2001) the proposed components of SELECT were summarized as including, "timely epidemiologic investigations of molecular and genetic markers that may contribute to the development of prostate, lung, colorectal, or other cancers ... based on 32,400 men aged 55 years or older (age 50 or older for the African-American men) enrolled into an intergroup, randomized, placebocontrolled, double-blind, phase III prevention trial of supplemental selenium [200 microgram Se as selenomethionine per day] and vitamin E [400 I.U. per day] developed and funded by the National Cancer Institute, and coordinated by the Southwest Oncology Group (SWOG)." As of May 6, 2004, SWOG had exceeded the 32,400 recruit goal but anticipated some ineligibles and will recruit through June 24, 2004 (personal communication).

In the meantime, data from the Clark trial were further analyzed through the end of the blinded treatment and provided a less exciting conclusion 
(Duffield-Lillico et al. 2003). Only participants who happened to start the trial with baseline plasma selenium concentrations in the lowest two tertiles had significant reductions in prostate cancer incidence which could be attributable to the Se supplement, and this was the source of the significant protective effect overall. [We note, however, that the mean plasma Se concentration at recruitment was reported as 114 microgram per L; an adequate Se status by world standards.] It can be argued that the Clark trial was stopped prematurely, and also that too much concern about the merits of "primary" compared with "secondary" endpoints was expressed. With respect to the Se dose of 200 microgram per day, SELECT will be more of the same, but the endpoints will be tidy! The enthusiasm for SELECT continues but an occasional commentator questioned the sufficiency of evidence to support such a large-scale prospective trial for patients who want to reduce the risk of prostate cancer (Moyad 2002).

\section{The target}

Every since the Clark et al. report in 1996, it seemed of more than passing interest to wonder about the Se status of the prostate gland. Accordingly, one would have thought that this would raise questions about the dose in any future trial, and would influence questions about mechanisms of possible protection from cancer. For example, is the prostate relatively low in selenium and thus a dietary supplement helps protect it from the insults of xenobiotics? Or, does the organ have a reasonable concentration of $\mathrm{Se}$, but extra Se inhibits the growth of precancerous cells more than healthy cells? Several indirect mechanisms could also be conceived, but knowing something about the prostate itself seemed essential.

It is remarkable that at the time of SELECT's activation in 2001 nothing was known about the concentration of Se in the target organ or whether any Se in a dietary supplement found its way to the prostate gland! Later, Arnold and Thrasher (2003) published the first values, which indicated that the prostate is reasonably well endowed with $\mathrm{Se}[1.32 \pm$ 0.09 microgram Se per g dry weight (range 1.24$1.42 ; \mathrm{n}=5$ ) and $0.214 \pm 0.012$ microgram Se per $\mathrm{g}$ wet weight (range 0.200-0.229)] and that the concentration in one subject [2.72 microgram Se per $\mathrm{g}$ dry weight and 0.421 microgram Se per $\mathrm{g}$ wet weight], who was the only one who later admitted to having undergone self-medication at 200 microgram Se per day, was about twice the average for the others. By surgical examination of the prostatectomy specimens, these samples (typically $0.6 \mathrm{~g}$ wet wt) from the peripheral zone were judged to be healthy tissue from a cancerous prostate. From one specimen the surgeon also removed a sample which was judged to be tissue from the transitional zone which exhibited benign prostatic hyperplasia $(\mathrm{BPH})$; this contained 1.50 microgram Se per g dry weight compared with 1.24 microgram Se per g dry weight in the healthy tissue from the same subject (Arnold and Thrasher 2003). Further comparisons will be required to establish any possible significance to this slight elevation.

We have consulted published reports, see especially Lyon et al. 1989, for other human organs at autopsy of accident victims, and have noted a range of $0.74-1.40$ microgram Se per g dry weight for kidney tissue, which was higher than for liver $(0.37-0.59)$, muscle $(0.13-0.22)$ or heart $(0.11-0.29)$. Thus our prostate gland range of 1.24-1.42 microgram Se per $\mathrm{g}$ dry weight is comparable to kidney and the prostate must now be considered reasonably well endowed with selenium.

Our benchmark values (Arnold and Thrasher 2003) on the human prostate were published in March 2003. Since that time one other set of analyses has been published. Gianduzzo et al., in September 2003, reported some measurements on tissue samples from transurethral resection of the prostate for $\mathrm{BPH}$. Sample size was not indicated nor was the concentration basis (dry or wet weight). [Private communication with one of the authors subsequently revealed that wet weight was involved.] These workers published values ranging around 0.196 microgram Se per $g$ (wet wt), which have questionable accuracy because of the small amounts of tissue supposedly involved, and also limited relevancy because of the lack of discrimination between healthy and BPH tissue in their samples. It happens to be close to our value of $0.214 \pm 0.012$ microgram Se per $\mathrm{g}$ wet wt (Arnold and Thrasher 2003). Gianduzzo et al. (2003) also indicated a small increase of $23 \%$ for samples from individuals previously taking a selenium dietary supplement of 200 microgram Se per day, for just one month. Again, their trend is more meaningful than the absolute values. Their value was also considerably less than our single observation (Arnold and Thrasher 2003) for healthy tissue, with a $100 \%$ increase.

\section{A note on concentration}

The accuracy and confidence attached to a particular concentration depend on both the amount of analyte which is measured and the amount of the tissue taken for analysis. Even extreme sensitivity in the chemical analysis is negated by tiny sample size. We raise this point because a remarkable number of published values on human tissues are compromised by error in the weight of sample, do not state whether dry or wet weight is the basis, and neglect to indicate the weight 
of tissue actually involved. In the case of wet weight estimates the loss of water from the specimen before sampling, and then lyophilization from frozen vials $\left(-80^{\circ} \mathrm{C}\right)$ with inappropriate caps, are but two of the problems which we have observed and minimized. We request about 0.6 gram wet weight of prostate tissue from the surgeon, store it on ice between surgery and laboratory, and weigh the sample in a closed, tared, plastic vessel as soon as possible (usually within 30 minutes). Likewise, it is important to develop a routine that takes samples to constant dry weight (Arnold and Thrasher 2003). The dry weight basis is the preferred standard.

\section{Selenium treatment level in SELECT and other trials}

The landmark trial by the Nutritional Prevention of Cancer Study Group (Clark et al. 1996,1998) involved a randomized trial of 200 microgram Se (as high Se brewer's yeast) daily, but only participants with baseline plasma selenium concentrations in the lowest two tertiles had significant reductions in prostate cancer incidence (Duffield-Lillico et al. 2003). The same daily supplement of 200 microgram Se (as selenomethionine) is now in SELECT and may not be high enough to demonstrate the full potential of Se against prostate cancer. Given that the prostate gland has one of the higher concentrations of selenium (Arnold and Thrasher 2003), a working hypothesis involving correction of a deficiency (e.g., in glutathione peroxidase) has now become less attractive than other suggestions including an inhibitory effect of elevated selenium on the survival of precancerous cells.

Other trials that are now underway include the Negative Biopsy Study in Arizona (Stratton et al. 2003a), which was initiated in 1999, wherein participants are randomized to receive zero, 200, or 400 microgram Se as selenium-enriched yeast tablets once daily. As of June 2003, 514 out of 700 anticipated subjects had been enrolled. Also, the related Watchful Waiting Study involves men receiving zero, 200, or 800 microgram Se as enriched yeast, once daily (Stratton et al. 2003b). The higher levels may afford much more impressive results than the preoccupation elsewhere with 200 microgram Se per day.

\section{Ethical issues}

NIH Publication No. 01-4978 (June 2001) advertises SELECT and informs prospective recruits, "We will give you two bottles of capsules. One will contain selenium or a placebo that looks like selenium. The other will contain vitamin $\mathrm{E}$ or a placebo that looks like vitamin E. You should take one capsule from each bottle every day. You don't need to change your diet. We will offer you and your significant other a free multivitamin that does not have selenium or vitamin E. If you are already taking supplements that contain selenium or vitamin E, we ask that you stop taking them."

SELECT contains 16,200 men (half the total) who receive a placebo rather than the 200 microgram Se treatment tablet and, if they have observed the recommendations of the study, they have quit any previous supplement, which is typically 20 microgram Se (and 45 I.U. vitamin E) in a one-a-day multiple vitamin and mineral tablet. Imposition of this restriction is obviously driven by the desire of the study directors to increase the power of the trial in exploring Se effects, but it is arguable that an alternative approach, namely the superimposition of treatment upon habitual diet and mineral supplement (if any) of the men, would have been more humane. [The same type of concern should apply to the vitamin $E$ aspect of SELECT.] Now the wives and significant others are asked to give up their previous supplements for seven to twelve years in order to avoid confusion at the kitchen table!

I have raised this issue for discussion during lectures in the U.S. and Australia, but have been disappointed by the paucity of audience concern. Remarks have included, "surely it won't do the women much damage to give up a supplement of 20 microgram $\mathrm{Se}$ and 45 I.U. vitamin $\mathrm{E}$ for 10 years!" On the other hand, some men who have become informed about the present options for prostate cancer prevention have elected not to participate in SELECT and to self-medicate. This is surely the bane of nutritional trials popular opinion that either giving up a supplement or trying a new one cannot do much harm, compared with "serious" medicine.

The environment for trials concerning prostate cancer has also been unsettling because of the background uncertainty of current screening. Data from series that lack randomization and controls are inadequate to determine benefit. The prostate, lung, colorectal and ovarian cancer screening trial of the National Cancer Institute, which will include 74,000 men (and 74,000 women) 60 to 74 years old, was initiated in 1993. The design includes testing the efficacy of using prostate specific antigen (PSA) and digital rectal examination (Gohagan et al. 1994).

A recent review (Thompson et al. 2004) stated that the optimum upper limit for the normal range of PSA is still unknown and concluded that biopsydetected prostate cancer, including high-grade cancers, is not rare among men with PSA levels of 4.0 nanogram per milliliter or less levels generally thought to be in the normal range. PSA testing in the U.S., which began in 1987, has become increasingly widespread and has led to a shift toward the identification of earlier-stage disease. The apparent improvement in better outcomes may be partly attributable to lead-time 
bias (i.e., the time gained by early diagnosis added to survival time). There is still insufficient evidence to state categorically that early detection by PSA screening can reduce morbidity or mortality: only randomized trials can answer this (Barry 2001).

A further frustration attends the current inability to identify an aggressive prostate cancer at early detection. Many are indolent as revealed by the present lifetime risk of diagnosis (about 16\%) compared with lifetime risk of death (about 3\%). A randomized trial in Scandinavia, comparing radical prostatectomy with watchful waiting in early prostate cancer, concluded that the former significantly reduced disease-specific mortality, but there was no significant difference with watchful waiting in terms of overall survival (Holmberg et al. 2002). The purpose of the Prostate Cancer Intervention Versus Observation Trial (PIVOT), initiated in 1994 by the Department of Veterans Affairs and the National Cancer Institute in the U.S., is likewise designed to determine which of these two strategies is superior in managing clinically localized prostate cancer over a 12 year follow-up period (Wilt and Brawer 1994).

\section{CONCLUDING REMARKS}

Some evidence supports the working hypothesis that supranutritional concentrations of Se afford protection from prostate cancer, and other data suggest that Se-deficiency may be associated with increased incidence of this and other cancers. However, cancer rates are no higher in people living in selenium-deficient areas of the world such as New Zealand. The onset of prostate disease is clearly associated with aging and yet there is no downward trend with age in the Se status of men, as measured by serum levels. Also, there is no evidence at hand to suggest that $\mathrm{Se}$ supplementation ameliorates symptoms or improves outcomes of benign prostatic hyperplasia or prostate cancer. With this background one of the biggest cancer prevention trials, SELECT, was initiated to see whether a daily supplement of 200 microgram Se (as selenomethionine), or 400 I.U. vitamin E, or both, would help men avoid prostate cancer. Questions about the appropriateness of the dose of Se have been raised in this review.

However, an even larger gap in this subject attends the target organ, the prostate gland. A small set of Se concentrations in the prostate has now been published. But there is obviously a need to accumulate a much larger number of measurements from histologically healthy tissue in the peripheral zone, from radical prostatectomies, and from healthy glands of accident victims at autopsy, and thereby establish normal ranges according to age and race. Also, analyses of cancer, benign prostatic hyperplasia, and healthy tissues from the same gland should be compared and examined for correlations with long-term Se status.

\section{ACKNOWLEDGMENT}

This study was supported by a grant to W.N.A. from the John and Effie E. Speas Memorial Trust of Kansas City, Missouri.

\section{REFERENCES}

Aaseth J., Smith-Kielland A., Thomassen Y.: Selenium, alcohol and liver diseases. Ann. Clin. Res. 18:43-47, 1986.

Arnold W.N., J.B. Thrasher: Selenium concentration in the prostate. Biol. Trace El. Res. 91:277-280, 2003.

Arthur J.R., Nicol F., Grant E., Beckett G.J.: The effects of selenium deficiency on hepatic type-I iodothyronine deiodinase and protein disulphide-isomerase assessed by activity measurements and affinity labeling. Biochem. J. 274:297-300, 1991.

Barry M.J.: Clinical practice. Prostate-specificantigen testing for early diagnosis of prostate cancer. N. Engl. J. Med. 344:1373-1377, 2001.

Beck M.A.: Rapid genomic evolution of a nonvirulent coxsackievirus B3 in seleniumdeficient mice. Biomed. Environ. Sci. 10:307315, 1997.

Beck M.A., Kolbeck P.C., Shi Q. et al.: Increased virulence of a human enterovirus (coxsackievirus B3) in selenium-deficient mice. J. Infect. Dis. 170:351-357, 1994.

Beck M.A., Shi Q., Morris V.C., Levander O.A.: Rapid genomic evolution of a non-virulent coxsackievirus B3 in selenium- deficient mice results in selection of identical virulent isolates. Nat. Med. 1: 433-436, 1995.

Brooks J.D., Metter E.J., Chan D.W. et al.: Plasma selenium level before diagnosis and the risk of prostate cancer development. J. Urol. 166:2034-2038, 2001.

Clark L.C., Cantor K.P., Allaway W.H.: Selenium in forage crops and cancer mortality in U.S. counties. Arch. Environ. Health 46:37-42, 1991.

Clark L.C., Combs Jr. G.F., Turnbull B.W. et al.: Effects of selenium supplementation for cancer prevention in patients with carcinoma of the skin. A randomized controlled trial. Nutritional Prevention of Cancer Study Group. JAMA. 276:1957-1963, 1996.

Clark L.C., Dalkin B., Krongrad A. et al.: Decreased incidence of prostate cancer with 
selenium supplementation: results of a doubleblind cancer prevention trial. Br. J. Urol. 81:730-734, 1998.

Duffield-Lillico A.J., Dalkin B.L., Reid M.E. et al.: Selenium supplementation, baseline plasma selenium status and incidence of prostate cancer: an analysis of the complete treatment period of the Nutritional Prevention of Cancer Trial. BJU Int. 91:608-612, 2003.

Epp O., Ladenstein R., Wendel A.: The refined structure of the selenoenzyme glutathione peroxidase at $0.2 \mathrm{~nm}$ resolution. Eur. J. Biochem. 133:51-69, 1983.

Ge K., Yang G.: The epidemiology of selenium deficiency in the etiological study of endemic diseases in China. Am. J. Clin. Nutr. 57:259263, 1993.

Gianduzzo T.R., Holmes E.G., Tinggi U. et al.: Prostatic and peripheral blood selenium levels after oral supplementation. J. Urol. 170:870873, 2003.

Gohagan J.K., Prorok P.C., Kramer B.S., Cornett J.E.: Prostate cancer screening in the prostate, lung, colorectal and ovarian cancer screening trial of the National Cancer Institute. J. Urol. 152:1905-1909, 1994.

Guilbert L.J., Iscoe N.N: Partial replacement of serum by selenite, transferrin, albumin and lecithin in haemopoietic cell cultures. Nature 263:594-595, 1976.

Hartman T.J., Taylor P.R., Alfthan G. et al.: Toenail selenium concentration and lung cancer in male smokers (Finland). Cancer Causes Control 13:923-928, 2002.

Hill K.E., McCollum G.W., Boeglin M.E., Burk R.F.: Thioredoxin reductase activity is decreased by selenium deficiency. Biochem. Biophys. Res. Commun. 234:293-295, 1997.

Holmberg L., Bill-Axelson A., Helgesen F. et al.: A randomized trial comparing radical prostatectomy with watchful waiting in early prostate cancer. N. Engl. J. Med. 347:781-789, 2002.

Hoque A., Albanes D., Lippman S.M. et al.: Molecular epidemiologic studies within the Selenium and Vitamin E Cancer Prevention Trial (SELECT). Cancer Causes Control 12:627-633, 2001.

Jensen R., Closson W., Rothenberg R.: Epidemiologic notes and reports: selenium intoxication New York. MMWR CDC Surveill. Summ. 33: 157-158, 1984. [see also, JAMA 251:1938, 1984.]

Kim S.P., Bennett C.L., Chan C. et al.: QOL and outcomes research in prostate cancer patients with low socioeconomic status. Oncology (Huntingt.) 13:823-832, 1999.

Klein E.A., Thompson I.M., Lippman S.M. et al.: SELECT: The selenium and vitamin $\mathrm{E}$ cancer prevention trial: rationale and design. Prostate cancer prostatic dis. 3:145-151, 2000.

Levander O.A.: Metabolic interrelationships and adaptations in selenium toxicity. Ann. N. Y. Acad. Sci. 192:181-192, 1972.

Levander O.A.: A global view of human selenium nutrition. Annu. Rev. Nutr. 7:227-250, 1987.

Levander O.A., Alfthan G., Arvilommi H. et al.: Bioavailability of selenium to Finnish men as assessed by platelet glutathione peroxidase activity and other blood parameters. Am. J. Clin. Nutr. 37:887-897, 1983.

Lombeck I., Kasperek K., Feinendegen L.E., Bremer H.J.: Serum-selenium concentrations in patients with maple-syrup-urine disease and phenylketonuria under diet-therapy. Clin. Chim. Acta 64:57-61, 1975.

Lyon T.D., Fell G.S., Halls D.J., Clark J., McKenna F.: Determination of nine inorganic elements in human autopsy tissue. J. Trace Elem. Electrolytes Health Dis. 3:109-118, 1989.

McKeehan W.L., Hamilton W.G., Ham R.G.: Selenium is an essential trace nutrient for growth of WI-38 diploid human fibroblasts. Proc. Natl. Acad. Sci. USA 73:2023-2027, 1976.

Medinsky M.A., Cuddihy R.G., Griffith W.C., McClellan R.O.: A stimulation model describing the metabolism of inhaled and ingested selenium compounds. Toxicol. Appl. Pharmacol. 59:54-63, 1981.

Moreno-Reyes R., Suetens C., Mathieu F. et al.: Kashin-Beck osteoarthropathy in rural Tibet in relation to selenium and iodine status. N. Engl. J. Med. 339:1112-1120, 1998.

Moyad M.A.: Selenium and vitamin E supplements for prostate cancer: evidence or embellishment? Urology 59 (4, suppl. 1):9-19, 2002.

Patterson E.L., Milstrey R., Stokstad E.L.R.: Effect of selenium in preventing exudative diathesis in chicks. Proc. Soc. Exptl. Biol. Med. 95:617620, 1957.

Pohl B., Schneider T.: Determination of Normal Levels of Selenium in Blood Serum by GFAAS. Varian Optical Spectroscopy Instruments, Instruments at work, AA-103, August 1991.

Raich P.C., Lü J., Thompson H.J., Combs G.F.: Selenium in cancer prevention: clinical issues and implications. Cancer Invest. 19:540-553, 2001.

Rotruck J.T., Pope A.L., Ganther H.E. et al.: Selenium: biochemical role as a component of glutathione peroxidase. Science 179:588-590, 1973.

Salonen J.T., Huttunen J.K.: Selenium in cardiovascular diseases. Ann. Clin. Res. 18:3035, 1986. 
Schaller K.H., Schiele R.: Selenium. In L. Alessio, A. Berlin, R. Roi (eds): Biological Indicators for the Assessment of Human Exposure to Industrial Chemicals, Comm. Eur. Commun., Luxembourg 1989, pp. 77-93.

Schwarz K., Foltz C.M.: Selenium as an integral part of Factor 3 against dietary necrotic liver degeneration. J. Amer. Chem. Soc. 71:32923293,1957.

Schwarz K., Bieri J.G., Briggs G.M., Scott M.L.: Prevention of exudative diathesis in chicks by Factor 3 and selenium. Proc. Soc. Exptl. Biol. Med. 95:621-625, 1957.

Shamberger R.J., Frost D.V.: Possible protective effect of selenium against human cancer. Can. Med. Assoc. J. 104:82-84, 1968.

Sheehan T.M.T., Halls D.J.: Measurement of selenium in clinical specimens. Ann. Clin. Biochem. 36:301-315, 1999.

Stadtman T.C.: Specific occurrence of selenium in enzymes and amino acid tRNAs. Faseb J. 1:375-379, 1987.

Stadtman T.C.: Biosynthesis and function of selenocysteine-containing enzymes. J. Biol. Chem. 266:16257-16260, 1992.

Stratton M.S., Reid M.E., Schwartzberg G. et al.: Selenium and prevention of prostate cancer in high-risk men: the Negative Biopsy Study. Anticancer Drugs 14:589-594, 2003 a.

Stratton M.S., Reid M.E., Schwartzberg G. et al.: Selenium and inhibition of disease progression in men diagnosed with prostate carcinoma: study design and baseline characteristics of the 'Watchful Waiting' Study. Anticancer Drugs 14:595-600, 2003b.
Sunde R.A.: Selenium. In B.L. O’Dell and R.A. Sunde (eds): Handbook of Nutritionally Essential Minerals, Marcel Dekker, New York 1997, pp. 493-556.

Thompson I.M., Pauler D.K., Goodman P.J. et al.: Prevalence of prostate cancer among men with a prostate-specific antigen level < or $=4.0 \mathrm{ng}$ per milliliter. N. Engl. J. Med. 350:2239-2246, 2004.

Vanderpas J.B., Contempre B., Duale N.L. et al.: Iodine and selenium deficiency associated with cretinism in northern Zaire. Am. J. Clin. Nutr. 52:1087-1093, 1990.

Willett W.C., Polk B.F, Morris J.S. et al.: Prediagnostic serum selenium and risk of cancer. Lancet. 2:130-134, 1983.

Wilt T.J., Brawer M.K.: The prostate cancer intervention versus observation trial: a randomized trial comparing radical prostatectomy versus expectant management for the treatment of clinically localized prostate cancer. J. Urol. 152:1903-1904, 1994.

Yang G., Zhou R.: Further observations on the human maximum safe dietary selenium intake in a seleniferous area of China. J. Trace Elem. Electrolytes Health Dis. 8:159-165, 1994.

Yang G., Wang S., Zhou R., Sun S.: Endemic selenium intoxication of humans in China. Am. J. Clin. Nutr. 37:872-881, 1983.

Yoshizawa K., Willett W.C., Morris S.J. et al.: Study of prediagnostic selenium level in toenails and the risk of advanced prostate cancer. J. Natl. Cancer Inst. 90:1219-1224, 1998 